\title{
Effects of ion motion on linear Landau damping
}

\author{
Hui $\mathrm{Xu}^{1 * *}$, Zheng-Ming Sheng ${ }^{2,3,4}, \mathrm{Xiang} \mathrm{Mu} \mathrm{Kong}^{1}$, Fu-Fang Su${ }^{1}$ \\ ${ }^{1}$ Shandong Provincial Key Laboratory of Laser Polarization and Information Technology, \\ Department of Physics, Qufu Normal University, Qufu 273165, China \\ ${ }^{2}$ SUPA, Department of Physics, University of Strathclyde, Glasgow G4 0NG, UK \\ ${ }^{3}$ Laboratory for Laser Plasmas and Department of Physics and Astronomy, Shanghai Jiao Tong \\ University, Shanghai 200240, China \\ ${ }^{4}$ Collaborative Innovation Center of IFSA, Shanghai Jiao Tong University, Shanghai 200240, \\ China
}

\begin{abstract}
The effects of ion motion on Landau damping has been studied by use of one-dimensional Vlasov-Poisson simulation. It is shown that the ion motion may significantly change the development of the linear Landau damping. When the ion mass is multiple of proton mass, its motion will halt the linear Landau damping at some time due to the excitation of ion acoustic waves. The latter will dominate the system evolution at the later stage and hold a considerable fraction of the total energy in the system. With very small ion mass, such as in electron-positron plasma, the ion motion can suppress the linear Landau damping very quickly. When the initial field amplitude is relatively high such as with the density perturbation amplitude $\delta n / n_{0}>0.1$, the effect of ion motion on Landau damping is found to be weak or even ignorable.
\end{abstract}

Keywords : Linear Landau damping, Ion motion, Vlasov-Poisson simulation

PACS: $52.35 . \mathrm{Fp}, 52.35 . \mathrm{Mw}, 52.35 . \mathrm{Sb}$

\footnotetext{
** Email: huixu3973@163.com
} 


\section{Introduction}

Landau damping is a key collective behavior of plasma discovered 70 years ago ${ }^{[1]}$. It is a kind of collisionless damping resulting from resonant energy exchange between an electrostatic wave and particles. In the linear regime when an electron distribution function (EDF) in a spatially uniform plasma is perturbed by a small-amplitude electrostatic wave, Landau's analysis predicts that the time-asymptotic evolution of the electric field exhibits exponential damping (or growing). The damping (or growing) rate $\gamma_{k}$ is proportional to the derivative of equilibrium EDF with respect to the velocity $v$ calculated at the phase velocity $v_{\mathrm{ph}}$ of the electrostatic wave. The treatment is rigorous when the wave amplitude is infinitesimal and the background ions are assumed to be immobile. The linear Landau damping has been well demonstrated in Vlasov-Poisson simulation by many researchers in the past decades ${ }^{[2-12]}$. This collisionless damping has become very important in a wide context (see reviews by Ryutov ${ }^{[13]}$ and Ivanov ${ }^{[14]}$ ) and some recent works show that progress in understanding and modeling this problem is still being made ${ }^{[15-22]}$. In these studies, the ions are usually assumed to be immobile as in the original theory ${ }^{[1]}$. However, ion motion can change the evolution of the plasma oscillations at certain time scale. For example, it has been shown that both ion motion and the relativistic effect of electron motion can lead to phase mixing phenomena if a plasma oscillation has moderate amplitude ${ }^{[23,24]}$. In an unstable nonlinear electrostatic system, it is found that ion motion can reduce the asymptotic growth of wave amplitudes ${ }^{[25,26]}$. In this paper, based upon Vlasov-Poisson simulations we show that the ion motion can even considerably change the linear Landau damping. In particular, it is found that the linear Landau damping will disappear at a certain stage of the electrostatic wave decay due to the excitation of ion acoustic waves.

\section{The model and governing equations}

For the simplest case, the electrostatic oscillations can be described with the one-dimensional Vlasov-Poisson model, which is govern by the following equations:

$$
\begin{gathered}
\frac{\partial f_{e}}{\partial t}+v_{e} \frac{\partial f_{e}}{\partial x}-E \frac{\partial f_{e}}{\partial v_{e}}=0 \\
\frac{\partial f_{i}}{\partial t}+v_{i} \frac{\partial f_{i}}{\partial x}+Z \frac{m_{e}}{m_{i}} \frac{\partial f_{i}}{\partial v_{i}}=0 \\
\frac{\partial E}{\partial x}=Z \int_{-\infty}^{+\infty} f_{i} d v_{i}-\int_{-\infty}^{+\infty} f_{e} d v_{e},
\end{gathered}
$$

where $f_{e}\left(x, v_{e}, t\right)$ and $f_{i}\left(x, v_{i}, t\right)$ are the distribution function of electrons and ions normalized by the equilibrium particle density $n_{0}$, respectively, and $E(x, t)$ is the electric field normalized by 
$\left(e / m_{e} \omega_{p e} c\right)^{-1}$. Here time $t$ is normalized to the inverse of the electron plasma frequency $\omega_{p e}^{-1}$, space coordinate $x$ to $c / \omega_{p e}$, velocity $v_{e}$ and $v_{i}$ to the speed of light $c, m_{\mathrm{i}}$ and $m_{e}$ are the rest mass of ions and electrons, respectively, and $Z$ is the charge number of the ions normalized to the electron charge $e$. The oscillations are excited by initializing a single Fourier mode $k_{m}$ with the following initial electron distribution,

$$
f_{e}\left(x, v_{e}, t=0\right)=\frac{1}{\sqrt{2 \pi} v_{\text {the }}}\left[1+\varepsilon \cos \left(k_{m} x\right)\right] \exp \left(-\frac{v_{e}^{2}}{2 v_{\text {the }}^{2}}\right),
$$

where $\varepsilon$ is the initial perturbation amplitude of the electron density, $k_{m}=2 \pi m / L$ is the wave number of the mode $m, L$ is the whole length of the system which is $2 \pi$ in all the following simulations, $v_{\text {the }}=\sqrt{K_{B} T_{e} / m_{e} c^{2}}$ is thermal velocity of electrons with the electron temperature $T_{e}$ and $K_{B}$ is Boltzmann's constant. Ions are initially uniform in the $x$-space and follow the Maxwellian distribution in the velocity space $f_{i}\left(x, v_{i}, t=0\right)=\left(\sqrt{2 \pi} v_{t h i}\right)^{-1} \exp \left(-v_{i}^{2} / 2 v_{t h i}^{2}\right)$, where $v_{t h i}=v_{t h e} \sqrt{T_{i} m_{e} / T_{e} m_{i}}$ is the normalized thermal velocity of ions with the ion temperature $T_{\mathrm{i}}$.

When solving the Vlasov equation, we use the time splitting method introduced by Cheng and Knorr ${ }^{[27]}$, which rests on splitting the Vlasov equation into separated spatial and velocity space advection equations. For each advection equation, we use the positive and flux conservative (PFC) method ${ }^{[28]}$. The fast Fourier transform method is used to solve the Poisson equation with the assumption of a periodic boundary in the longitudinal space.

\section{Numerical simulation results}

\subsection{Disappearance of linear Landau damping}

In the following simulations, we keep a fixed wave number $k_{m}=1$. The electron thermal velocity is $v_{\text {the }}=0.4$, the ion mass is normalized by electron mass $m_{\mathrm{e}}$ by defining $m_{\mathrm{e}}=1, m_{\mathrm{i} 0}=m_{\mathrm{i}} / \mathrm{m}_{\mathrm{e}}$. In these simulations $m_{\mathrm{i} 0}=1836=m_{\mathrm{p}}$ (here $m_{\mathrm{p}}$ is the mass of a proton), the ion temperature is equal to the electron temperature $\left(T_{i}=T_{e}\right)$ and the ion charge $Z=1$. Figure 1 shows how the fundamental mode of the electric field evolves with time under different initial amplitudes of the wave. When the initial perturbation amplitude is high such as $\varepsilon=0.1$ shown in Fig. 1(a), the system is highly nonlinear. It indicates that the systems evolve basically identical either with or without ion motion in this case, i.e., ion motion does not lead to obvious change of the field evolution when the initial 
amplitude is large. However, if the initial amplitude is as small as near the linear region, the effect of ion motion becomes obvious, as shown in Figs. 1(b)-1(d). It shows that the linear Landau damping develops at the beginning. From about $t=61$, the field evolution starts to deviate from the linear Landau damping. From the time $t=100$, the Landau damping almost disappears. Instead, the field evolution appears to decay slowly with a large periodic structure. The period of the field evolution is as long as about $\Gamma=340 \omega_{p e}^{-1}$, which is about 65 multiples of the period of the electron plasma wave governed by the dispersion relation $\omega^{2}=\omega_{p e}^{2}+3 k^{2} v_{t h e}^{2} / 2$. This large structure should be associated with ion motion or the excitation of ion acoustic waves. The frequency of the ion acoustic wave is given by

$$
\omega_{i}^{2}=\gamma_{i} k^{2} v_{t h i}^{2}+Z \gamma_{e} k^{2} v_{t h i}^{2}\left(1+\gamma_{e} k^{2} \lambda_{D e}^{2}\right)^{-1}\left(T_{e} / T_{i}\right)
$$
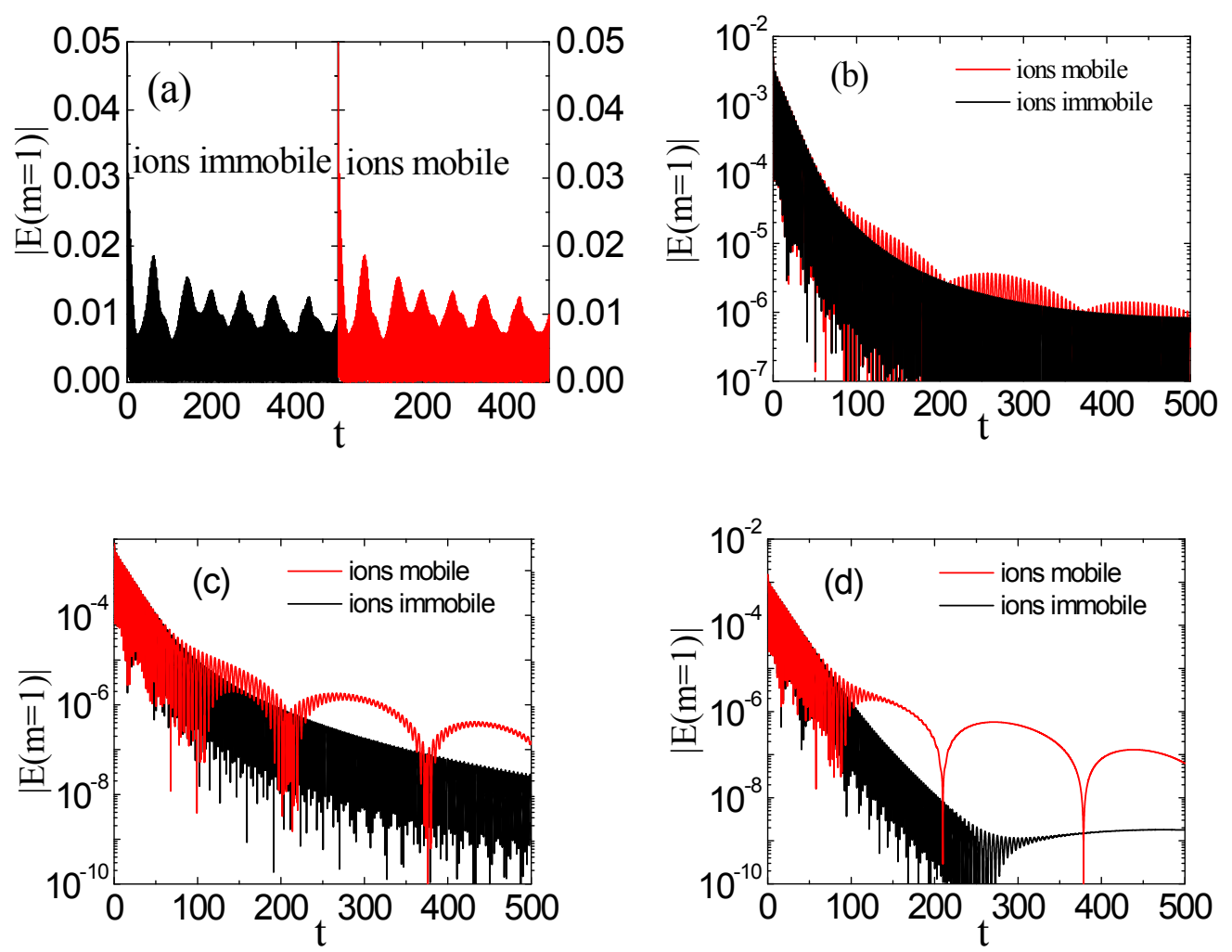

Figure 1 Time evolution of the electric field fundamental mode under different initial amplitudes: (a) $\varepsilon=0.1$, (b) $\varepsilon=0.01$, (c) $\varepsilon=0.008$, (d) $\varepsilon=0.003$, here $v_{\text {the }}=0.4$, $m_{\mathrm{i} 0}=1836=m_{\mathrm{p}}, T_{\mathrm{e}}=T_{\mathrm{i}}$, and $k_{\mathrm{m}}=1$.

For the given parameters in the simulation and with the adiabatic constants in the one-dimensional situation $\gamma_{i}=\gamma_{e}=3$, one finds the frequencies of electron plasma wave and ion acoustic wave are $\omega=1.21655 \omega_{p e}$ and $\omega_{1}=0.02093 \omega_{p e}$, respectively. Therefore, the ion acoustic cycle is about 58 multiples of the electron plasma wave cycle, which is a little shorter than the cycle of the 
slowly decaying structure in the simulation results shown in Fig. 1. This difference may be caused by the plasma wave phase velocity shift when the ion motion is included, as discussed later.
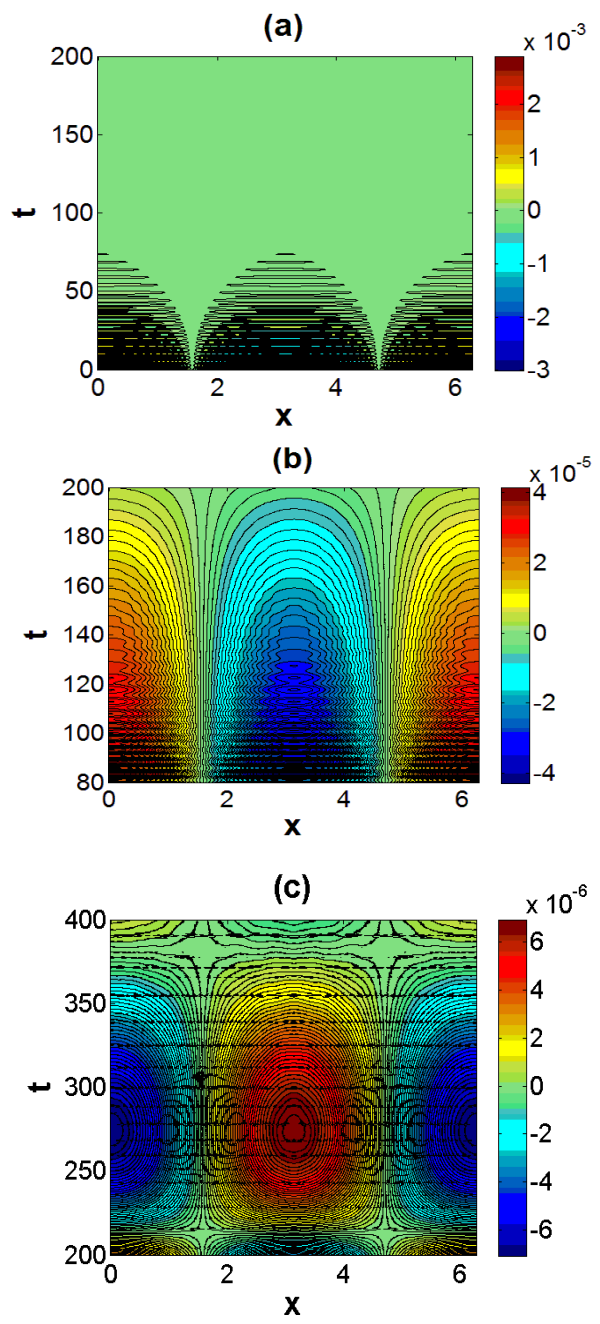

Figure 2 Time evolution of electron density perturbation $\delta n_{e}$ with the ion motion included during $t=[0,200]$ (a), [80,200] (b), and [200,400] (c), where the other parameters are the same as those in Fig. 1(d).

Figures 2 and 3 illustrate the time evolution of electron density perturbation amplitude $\delta n_{e}$ and ion density perturbation amplitude $\delta n_{i}$, respectively, in different time periods for $\varepsilon=0.003$. Figure 2(a) shows that the amplitude of the longitude electron plasma wave is damped to very small level at about $t=80$. On the contrary, the amplitude of the ion acoustic wave increases with the time and reaches the maximal value at about this moment $t=80$ [see Fig. 3 (a)]. As shown in Figs. 3(c) and 3(d), the ion density perturbation becomes at the same level as the electron density perturbation at the time $t=70$. After this time, the electron plasma wave undergoes further damping and the ion acoustic wave starts to dominate the evolution of the plasma system. Electrons in the system mainly follow the ion motion in the later period as shown in Figs. 2(c) and 3(b). Therefore, the slow electric field oscillations in the later stage correspond to the ion acoustic 
wave frequency as shown in Fig. 1(d). In passing, we point out that ion density perturbations can also develop when the initial electron plasma wave is high (such as $\varepsilon=0.1$ ). However, the electron density perturbations are always much larger than the excited ion density perturbations. So the electron dynamics remains predominant during the whole evolution of the system, i.e., the ion mobile effect on the field evolution is very small and can be neglected when the initial perturb amplitude is large.
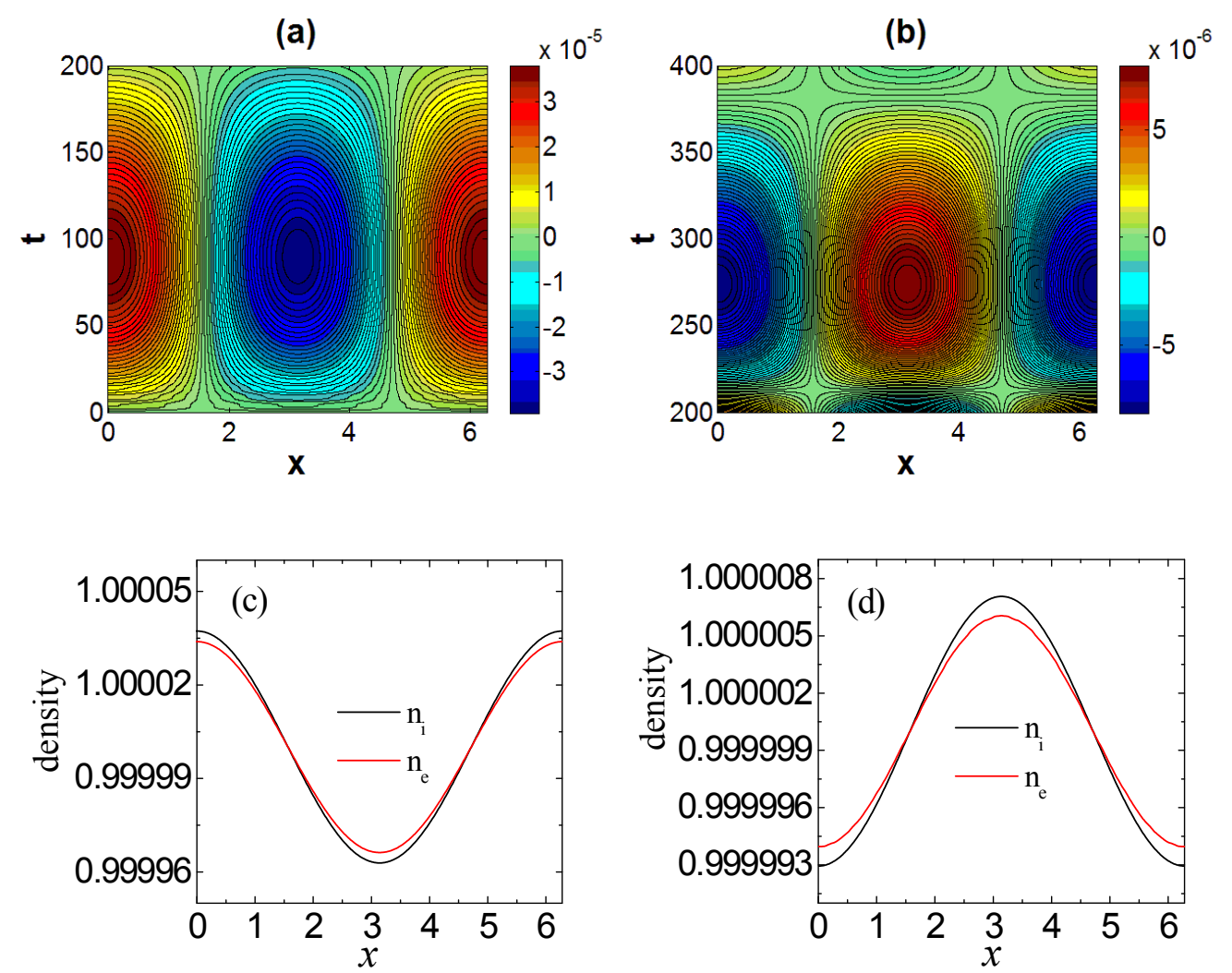

Figure 3 Time evolution of ion density perturbation $\delta n_{i}$ during $t=[0,200]$ (a) and [200,400] (b), where the other parameters are the same as those in Fig. 1(d). The corresponding snapshots of electron and ion density distributions in the $\mathrm{x}$-space at the time $t=70$ and $t=250$ are also shown in (c) and (d), respectively.

\subsection{Effect of ion mass}

To show more explicitly effect of ion motion, time evolution of the electric field fundamental mode with different ion mass is shown in Fig. 4. With the increase of the ion mass, the influence of ion motion on the linear Landau damping occurs later with larger oscillation periods accordingly. Most of the electric field energy has been damped to the particles with velocity near the phase velocity. We also have calculated time evolution of the electric field fundamental mode 
with other different ion charge and different ion mass. With the increase of the ion charge, the time that ion mobile begins to influence the linear Landau damping $\tau_{d}$ is early when the ion mass is the same. The period of stimulated acoustic wave $\Gamma_{i}$ is almost the same as long as the ion charge to mass ratio is the same, which is in accordance with theoretical scaling for ion acoustic period $\Gamma_{i} \propto \sqrt{m_{i 0} / Z}$. For the heavier ions $\left(m_{\mathrm{i} 0}>200 m_{\mathrm{p}}\right)$, the time $\tau_{d}$ is almost the same when the ion mass and the ion charge increase the same multiple
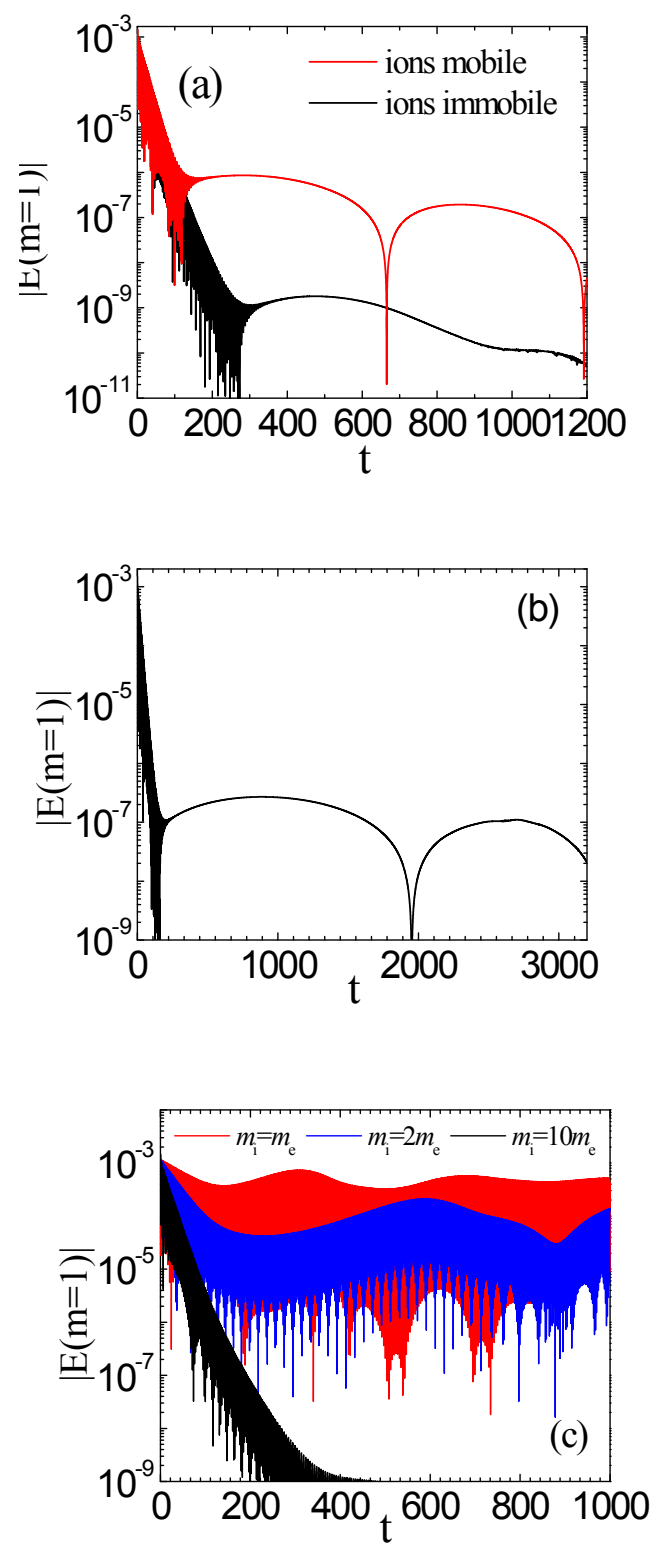

Figure 4 Time evolution of the electric field fundamental mode under different ion mass with $\mathrm{Z}=1$, (a) $m_{\mathrm{i} 0}=10 m_{\mathrm{p}}=18360$, (b) $m_{\mathrm{i} 0}=100 m_{\mathrm{p}}$, and (c) $m_{\mathrm{i} 0}=1,2$, and 10. The other parameters are same as those in Fig. 1(d). 
When the ion mass is so small such as comparable to the electron mass, Fig. 4(c) shows time evolution of the electric field fundamental mode with different light ion mass. When the normalized ion mass $m_{\mathrm{i} 0}=10$, the linear Landau damping can still be found clearly. However, when $m_{\mathrm{i} 0}=1$ or 2 , the field damping can only exist at the very early stage with a reduced damping rate. From Fig. 5 it can be seen the ion density perturbation can be excited very quickly (at about $t=1.9$ or about half of an electron oscillation period) when the ion mass equal to electron mass (i.e., electron-positron plasma). Afterwards, the system evolution is mainly dominated by energy exchange between electrons and positrons via electric fields.

(a)
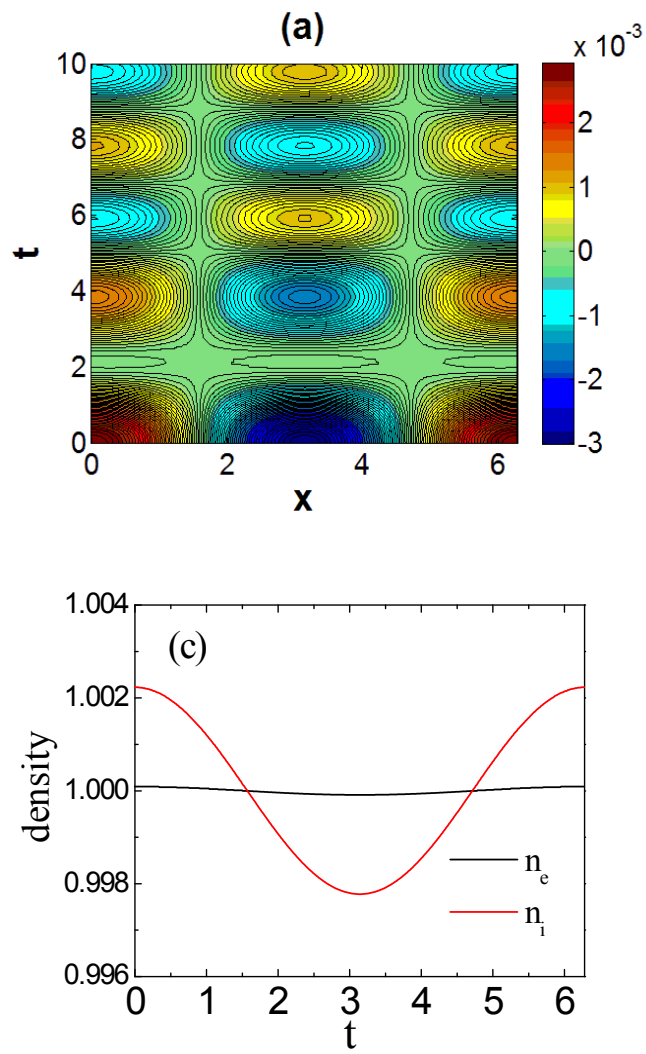

(b)
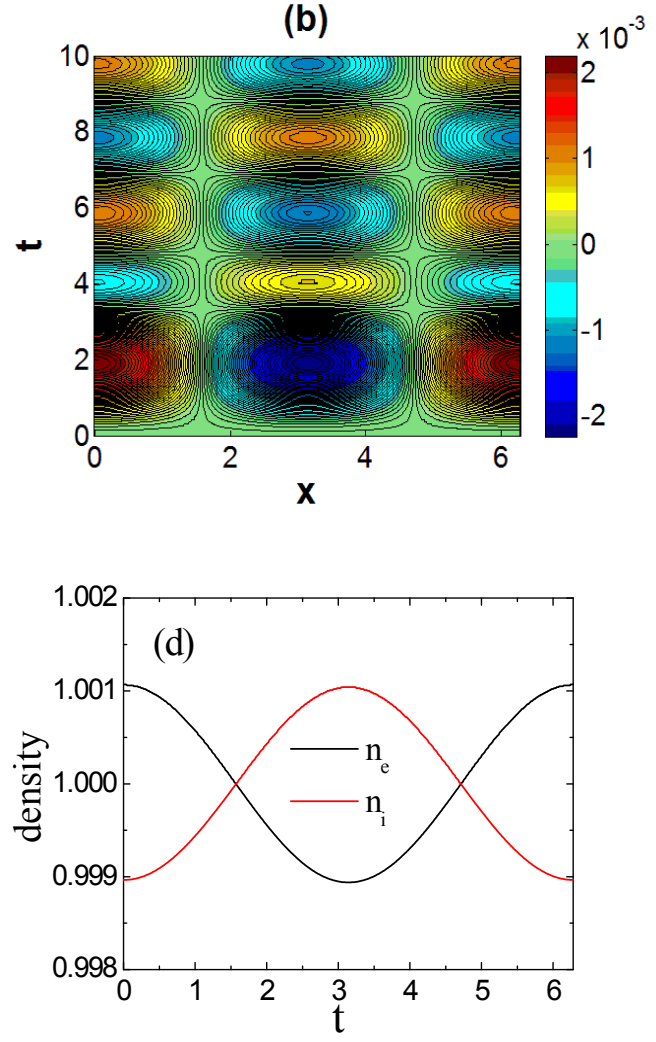

Figure 5 Time evolution of electron density perturbation $\delta n_{e}$ (a) and ion density perturbation $\delta n_{i}$ (b) at the early stage when $m_{i 0}=1$ (that is for positron), (c) and (d) show snapshots of the electron and positron density at $t=1.9$ and 7.8, respectively. Here $m_{\mathrm{i} 0}=1$, the other parameters are the same as those in Fig. 1(d).

In order to explain the field evolution in electron-positron plasma, we calculate the averaged distribution of the electrons and ions in $\mathrm{x}$-space, that is 


$$
\left\langle f_{e, i}\left(v_{e, i}\right)\right\rangle=L^{-1} \int_{0}^{L} f_{e, i}\left(x, v_{e, i}\right) d x .
$$

With the damping of the electric field, some particles with velocity slightly lower than the wave phase velocity absorb energy from the wave and can be accelerated. As the differential coefficient absolute value of the electron distribution $\left|d f_{e} / d v_{e}\right|$ is small at the wave phase velocity, the differential coefficient can change its sign very easy to satisfy $\left|d f_{e} / d v_{e}\right|>0$, which can be found in Fig. 6(a). After that, there are more particles having velocities slightly greater than the wave phase velocity which will be decelerated and losing energy to the wave. When the ion motion is included, the averaged distribution in $v$-space of electron distribution and ion distribution are always the same in the electron-positron plasma due to their same mass and same charge number [see Fig. 6(b)]. Figure 6(c) compares the averaged distribution of electrons either with or without the positron motion at $t=20$. The differential coefficient of electron distribution with the positron motion evolves much slower than those without the positron motion. This can explain why the ion motion can decrease the damping rate of the electric field fundamental mode. Compare Figs. 6(a) and 6(b), it can be seen that the resonance domain in $v$-space is moved to a larger velocity direction when the ion motion is considered. This implies the phase velocity increase. According to the formula $v_{p h}=\omega / k$, it can be deduced that the electron plasma wave frequency increases and oscillation period decreases when the positron motion is considered. From Fig. 6(d) it is shown that the plasma wave frequency increases when the positron motion is considered, which is in accordance with the theory analysis ${ }^{[29,30]}$. And this is found to be true for ions taking other mass ratio although the plasma wave phase velocity shift is not as obvious as in the positron-electron plasma the ion mass is large. This can explain why the ratio of the ion acoustic wave period to the electron plasma wave period found from simulation shown in Fig. 1 is a little larger than the theoretical value where the ion motion is not considered. 

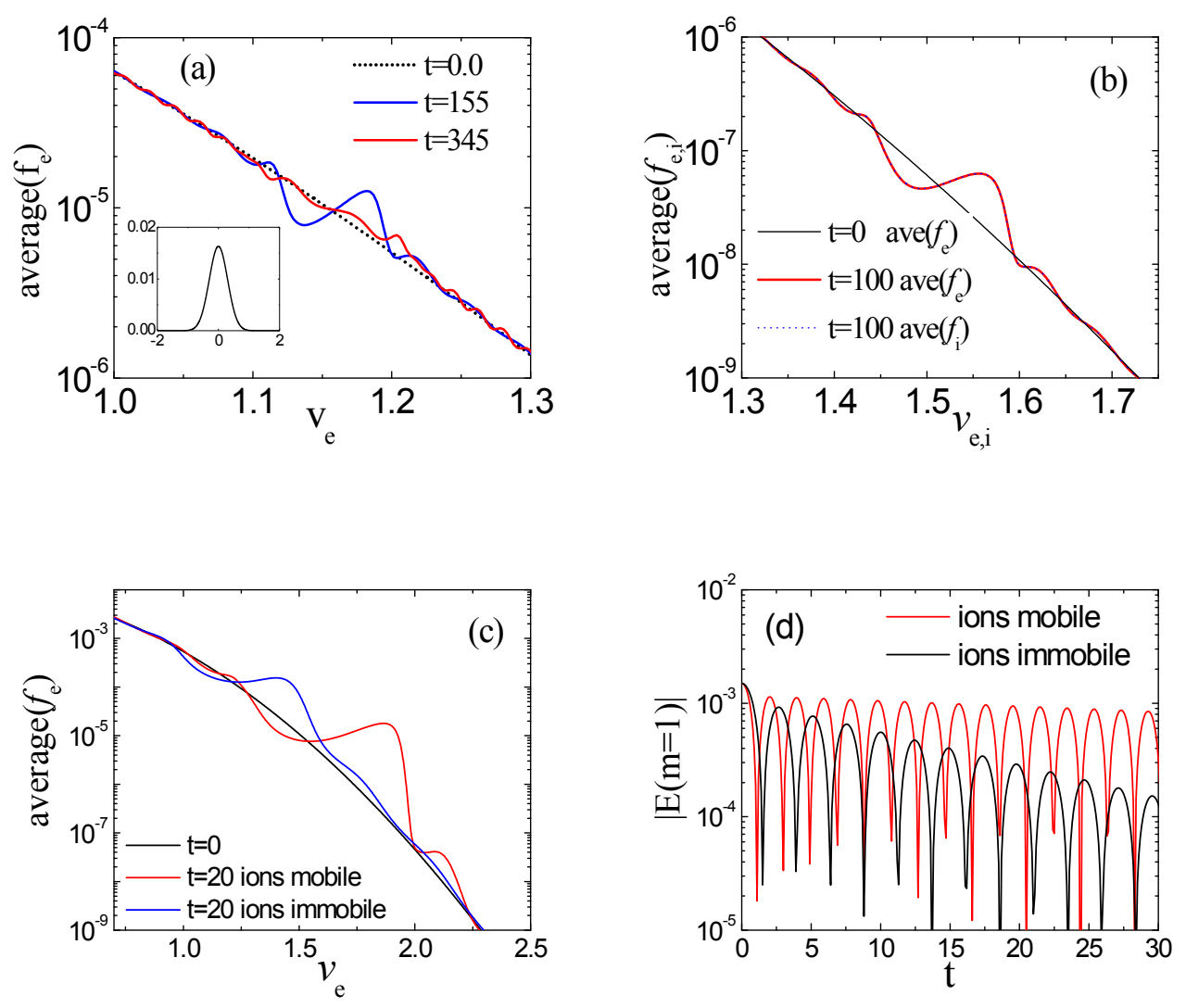

Figure 6 Averaged distribution of electrons and positrons in $\mathrm{x}$-space, (a) $v_{\text {the }}=0.3$, immobile positrons, (b) $v_{\text {the }}=0.3$, positrons mobile, (c) $v_{\text {the }}=0.4$, (d) evolution of the electric field fundamental mode at the early stage when $v_{\text {the }}=0.6$. The other parameters are same as those in Fig. $6(\mathrm{~d})$ with $m_{\mathrm{i} 0}=1$, that is electron-positron plasma.

\section{Conclusions}

Using one-dimensional Vlasov-Poisson simulation, we have studied the effect of ion motion on Landau damping. In the linear regime of Landau damping with a small initial amplitude of electron plasma waves, it is shown that the excited ion acoustic wave can significantly affect the system evolution. When the ion mass is multiple of proton mass, its motion will stop the linear Landau damping at some time and the excited ion acoustic wave will dominate the system evolution at the later stage. As a result, the residual field energy with ion motion will be much larger (such as 100 times) than that without considering ion motion. With small ion mass, such as in electron-positron plasma, the ion motion significantly suppresses the linear Landau damping rate and the system evolution is mainly dominated by energy exchange between electrons and positrons via electric fields. When the initial density perturbation is large in the quasi-nonlinear 
regime, the effect of ion motion becomes weak and the system is mainly determined by the electron dynamics.

\section{Acknowledgement}

This work is supported by the National Natural Science Foundation of China (Grant No. 11147025, 11275112, 11374210, 11421064, and 11220101002). ZMS acknowledges the support of the support of a Leverhulme Trust Research Project Grant at the University of Strathclyde.

\section{REFERENCES}

[1] L. D. Landau, J. Phys. (Moscow) 10, 25 (1946).

[2] J. Klimas, J. Comput. Phys. 68, 202 (1987).

[3] A. Harten and S. Osher, SIAM J. Numer. Anal. 24, 279 (1987).

[4] A. Ghizzo, P. Bertrand, M. Shoucri, T. W. Johnston, E. Filjakow and M. R. Feix, J. Comput. Phys. 90, 431 (1990).

[5] J. Klimas and W. M. Farrell, J. Comput. Phys. 110, 150 (1994).

[6] T. Utsumi, T. Kunugi, and J. Koga, Comp. Phys. Commun. 108, 159 (1998).

[7] E. Sonnendrücker, J. Roche, P. Bertrand, and A. Ghizzo, J. Comput. Phys. 149, 201 (1998).

[8] T. Nakamura and T. Yabe, Comput. Phys. Commun. 120, 122 (1999).

[9] E. Fijalkow, Comput. Phys. Commun. 116, 319 (1999).

[10] E. Sonnendrücker, J. J. Barnard, A. Friedman, and D. P. Grote, and S. M. Lund, Nuclear Instruction and Method in Physics Research A 464, 653 (2001).

[11] F. Filbet and E. Sonnendruecker, Comput. Phys. Commun. 150, 247 (2003).

[12] H. Xu and Z. Sheng, Plasma Sci. Tech. 14, 181 (2012).

[13] D. D. Ryutov, Plasma Phys. Controlled Fusion 41, A1 (1999).

[14] A. V. Ivanov, I. H. Cairns, and P. A. Robinson, Phys. Plasmas 11, 4649 (2004).

[15] M. C. Firpo, F. Doveil, Y. Elskens, P. Bertrand, M. Poleni, and Guyomarc'h, Phys. Rev. E 64, 026407 (2001); F. Doveil, M.-C. Firpo, Y. Elskens, D. Guyomarc'h, M. Poleni, and P. Bertrand, Phys. Lett. A 284, 279 (2001).

[16] Z. Sedláček and L. Nocera, Phys. Lett. A 296, 117 (2002).

[17] J. R. Danielson, F. Anderegg, and C. F. Driscoll, Phys. Rev. Lett. 92, 245003(2004).

[18] E. W. Laing and D. A. Diver, Phys. Plasmas 13, 092115(2006).

[19] V. Bratanov, F. Jenko, D. Hatch, and S. Brunner, Phys. Plasmas 20, 022108 (2013). 
[20] J. D. Callen, Phys. Plasmas 21, 052106 (2014).

[21] J. Wesson, Phys. Plasmas 22, 022519 (2015).

[22] B. Young, J. Math. Phys. 57, 021502 (2016).

[23] H. Xu, Z. M. Sheng, and J. Zhang, Phys. Scr. 74, 673 (2006).

[24] H. Xu, Z.M. Sheng, and J. Zhang, Acta Phys. Sinica 56, 968 (2007) (in Chinese).

[25] J. D. Crawford, Phys. Plasmas 2, 97 (1995).

[26] J. D. Crawford and A. Jayaraman, Phy. Rev. Lett. 77, 3549 (1996).

[27] C. Z. Cheng and G. Knorr, J. Comput. Phys. 22, 330 (1976).

[28] F. Filbet, E. Sonnendrücker, and P. Bertrand, J. Comput. Phys. 172, 166 (2001).

[29] N. Iwamoto, Phys. Rev. E 47, 604 (1993).

[30] G. P. Zank and R. G. Greaves, Phys. Rev. E 51, 6079 (1995). 